\title{
Métrica alternativa para dados governamentais abertos na América Latina
}

\author{
Alternative metric for open government data \\ in Latin America
}

\author{
Patrícia NASCIMENTO-SILVA' ${ }^{1}$ (D) 0000-0002-2405-8536 \\ Marta Macedo KERR-PINHEIRO2 iD 0000-0001-5592-3396
}

\section{Resumo}

O objetivo deste artigo é, a partir da DGABr, que configura uma métrica alternativa criada em tese de doutorado de 2018, mensurar os dados governamentais abertos de outros países da América Latina. O objetivo geral foi subdivido em três objetivos específicos: (1) identificar os países da América Latina que possuem legislação sobre acesso à informação/política de dados abertos definida; (2) identificar as legislações mais abrangentes e com maiores critérios de avaliação dos dados governamentais abertos; e (3) analisar a DGABr com base em outras legislações a fim de propor adequações para sua utilização em outros países. Nesse sentido, investigaram-se as legislações de acesso à informação dos países da América Latina a partir do ranking Global Right to Information Rating, que avalia as legislações de acesso à informação a nível mundial. A legislação do México foi escolhida como referência para comparação com a DGABr, pois atingiu, no ranking, a maior pontuação da América Latina. Analisando a legislação mexicana, foram identificados itens a serem incluídos na DGABr e também muitas semelhanças que já são avaliadas pela DGABr. Conclui-se que a DGABr permite adaptações e pode ser empregada em diferentes contextos e países da América Latina.

Palavras-chave: Dados Governamentais Abertos. DGABr. Dados Abertos. Métricas alternativas.

\begin{abstract}
The objective of this article is to use the DGABr, an alternative metric created, in a doctoral thesis of 2018, to evaluate the Open Government Data of Brazil, to measure the open government data of other Latin American countries. The overall objective was subdivided into three specific objectives: (1) Identify the countries of Latin America that have defined access to information/open data policy legislation; (2) Identify the most comprehensive legislation and criteria for assessing open government data; and (3) Analyze the DGABr based on other legislation and propose adjustments for its use in other countries. In this sense, the legislation on access to

\footnotetext{
1 Universidade Federal de Minas Gerais, Centro de Computação, Divisão de Aplicativos e Sistemas. Av. Antônio Carlos, 6627, Pampulha, 31270-901, Belo Horizonte, MG, Brasil. Correspondência para/Correspondence to: P. NASCIMENTO-SILVA. E-mail: <patricia.inf@gmail.com>.

2 Universidade Fumec, Faculdade de Ciências Empresariais, Programa de Pós-Graduação em Sistemas de Informação e Gestão do Conhecimento. Belo Horizonte, MG, Brasil.

Artigo elaborado a partir da tese de P. NASCIMENTO-SILVA (2018), intitulada "Dados Governamentais Abertos: métricas e indicadores de reúso". Universidade Federal de Minas Gerais, 2018.

Recebido em 18 de fevereiro de 2019, versão final reapresentada em 29 de maio de 2019 e aprovado em 25 de junho de 2019.
}

Como citar este artigo/How to cite this article

Nascimento-Silva, P.; Kerr-Pinheiro, M.M. Métrica alternativa para dados governamentais abertos na América Latina. Transinformação, v.31, e190009, 2019. http://dx.doi.org/10.1590/2318-0889201931e190009 
information of Latin American countries was investigated, based on the Global Right to Information Rating ranking, which assesses legislation on access to information worldwide. The legislation of Mexico was chosen as reference for comparison with the DGABr, having reached in the ranking, the highest score in Latin America. Analyzing Mexican legislation, items to be included in the DGABr were identified and many similarities were already evaluated by the DGABr. It is concluded that the DGABr allows adaptations and can be used in different contexts and countries of Latin America.

Keywords: Open Government Data. DGABr. Open Data. Alternative metrics.

\section{Introdução}

O acesso à informação na América Latina possui iniciativas recentes, as quais são apoiadas em experiências mundiais iniciadas desde 2006, quando a Corte Interamericana dos Direitos Humanos reconheceu que o direito de acesso à informação pública é um direito humano fundamental. Em 2008, foram criados os 10 princípios sobre o direito de acesso à informação, que foram declarados pelo Comitê Jurídico Interamericano (CJI) da Organização dos Estados Americanos (OEA) (Perlingeiro; Díaz; Liani, 2016).

Em 2010, a OEA criou a Lei Modelo de Acesso à Informação para países das Américas. Ela estabeleceu padrões mínimos, isto é, um ponto de referência para os países criarem suas próprias leis (Brasil, 2018).

A Colômbia foi um dos primeiros países a promulgar uma ampla lei de liberdade de informação. A lei foi promulgada em 1985, muito antes da maioria das democracias estabelecerem essas medidas. A "lei colombiana 57", como é conhecida, foi substancialmente reforçada por emendas em 2011 e, novamente, em 2014.

A Lei Federal sobre Transparência e Acesso à Informação Pública do México, promulgada em 2002, foi considerada modelo para legislação sobre transparência e acesso à informação, tanto em seu âmbito legal como em suas ferramentas de consecução. Ela incluiu recursos próprios suficientes e uma autoridade autônoma para o Instituto Nacional de Acesso à Informação, fundado para monitorar a aplicação da lei. A lei do México é qualificada como a melhor do mundo no índice das leis nacionais de liberdade de informação compiladas pelo Centro de Direito e Democracia (Organización de las Naciones Unidas para la Educación, 2017).

As legislações do Chile (2008) e do Brasil (2011) são as mais recentes e se beneficiaram do exemplo colombiano e mexicano, bem como de outros exemplos internacionais, incluindo a histórica Constituição Sul-Africana de 1996 e a Lei de Liberdade de Informação dos Estados Unidos da América de 1966. México e Brasil oferecem abordagens legais inovadoras para governos nacionais organizadas pelo governo federal, com acesso a leis de informações apoiadas por garantias constitucionais e aplicáveis aos governos estaduais e municipais, bem como a ministérios e agências federais.

Diferentemente, o mesmo não ocorre com as leis de outros sistemas federais, como os Estados Unidos, onde a Lei de Liberdade de Informação de 1966 só se aplica ao poder executivo do governo nacional. As leis vigentes que garantem o acesso à informação no México, no Chile, no Brasil e no Uruguai foram mais detalhadas e possuem maior alcance, com aplicabilidade digital e legal em todos os níveis do governo, nacional e local. Com exceção da Bolívia, Costa Rica, Cuba e Venezuela, quase todos os países da América Latina têm leis que garantem o acesso à informação oficial (Organización de las Naciones Unidas para la Educación, 2017).

Os dados abertos constituem um domínio da ciência aberta ainda recente, principalmente para os países da América Latina. As políticas instituídas nesses países são novas e estão em construção. Os Dados Governamentais Abertos (DGA) são um subdomínio dos dados abertos e referem-se aos dados do setor público, tornando a informação livremente disponível em formatos abertos e permitindo vias de acesso públicas que facilitem a sua exploração (Kalampokis, Tambouris, Tarabanis, 2011).

Os DGA representam um potencial recurso para organizações, indivíduos e para o próprio governo, que pode utilizá-los para melhorar seus conjuntos de dados, para o desenvolvimento de novos produtos ou mesmo 
para trazer inovações em seus serviços, com ganho de eficiência. Além disso, promove transparência, colaboração, participação e valores sociais e econômicos (Nascimento Silva; Kerr Pinheiro, 2018).

Diante desse contexto e após as iniciativas de governo aberto, protagonizadas pelos Estados Unidos da América (EUA) em 2009, pelas publicações do W3C sobre governo eletrônico e pelas revisões das legislações sobre abertura de dados pela comunidade europeia, como a adoção da Directiva 2003/98/CE, vários países desenvolveram ações, planos e políticas de dados abertos para estimular a reutilização de informações do setor público (Simón et al., 2012). No entanto, o movimento de abertura de dados e o acesso à informação somente será "completo" a partir do momento em que os dados disponibilizados cumprirem com seus objetivos e estiverem em conformidade com as legislações e políticas de dados abertos nas quais estão submetidos. Dessa forma, é necessário pensar à frente para avaliar o retorno do investimento feito na disponibilização dos DGA, principalmente em relação aos formatos de publicação de dados e o seu reúso (Nascimento Silva; Kerr Pinheiro, 2017).

A falta de padrões para avaliar a estrutura e o conteúdo fornecido em portais de dados dificulta a avaliação dessas iniciativas, uma vez que os autores empregam diferentes abordagens. Nesse sentido, ampliam-se os questionamentos na literatura ao mesmo tempo em que parece haver um aumento crescente de interesse em práticas de benchmarking para DGA (Lowry, 2015).

Na pesquisa realizada por Nascimento Silva e Kerr Pinheiro (2018), foram identificados padrões internacionais para publicação de dados abertos e seis metodologias principais (modelos e métricas) para mensurar dados abertos e DGA: MePOD-VS, Meloda, Norma UNE 178301, Open Data Maturity Model (ODMM), Open Data Maturity Model (Open Data Institute) e OpenGovB. Foi observado que as métricas refletem o resultado das políticas de informação e de seu subdomínio de dados abertos. Além disso, destaca-se que as métricas encontradas são recentes e ainda pouco utilizadas. Contudo, seu emprego, naturalmente alavancado pelos países que já conduzem o processo, irá gerar um maior grau de maturidade (Nascimento Silva; Kerr Pinheiro, 2018).

Diante da importância de mensurar os DGA e da ausência de métricas para avaliação dos dados disponibilizados pelo Governo Brasileiro, a DGABr (Dados Governamentais Abertos do Brasil) é uma métrica alternativa criada em 2018, como resultado de uma pesquisa de doutorado. Ela é baseada em experiências internacionais para avaliar os DGA disponibilizados pela Administração Pública Federal (APF) do Brasil. A DGABr foi construída com base em três pilares: (1) Legislação brasileira sobre DGA; (2) Experiência brasileira no reúso dos DGA publicados; e (3) Métricas, modelos e padrões internacionais para publicação de DGA. A DGABr, em sua primeira versão, mostrouse viável ao contexto brasileiro durante o estudo realizado e apresentou resultados positivos (Nascimento Silva, 2018a).

Considerando que os países da América Latina tendem a seguir padrões semelhantes, muito deles oriundos de boas práticas e recomendações de organizações regionais como a OEA e das possíveis semelhanças em relação ao acesso à informação, a questão que norteia este artigo é: A DGABr pode ser adaptada para o contexto da América Latina? Com esse questionamento, o artigo tem o objetivo geral de ampliar a abrangência da métrica DGABr em sua aplicabilidade a outros países da América latina, a partir de legislações de sucesso.

O objetivo geral da pesquisa foi subdividido em três específicos: (1) identificar os países da América Latina que possuem legislação sobre acesso à informação/política de dados abertos definida; (2) identificar as legislações mais abrangentes e com maiores critérios de avaliação dos DGA; e (3) analisar a DGABr com base em outras legislações a fim de propor adequações para sua utilização na América Latina.

A existência de poucas métricas para avaliação dos dados abertos é uma das motivações deste estudo, que faz uma adaptação da métrica DGABr a fim de compartilhar a pesquisa realizada para a construção da métrica brasileira. Ademais, é sugerida a sua utilização aos países da América Latina, que apesar de possuírem contextos diferentes, podem partilhar padrões em comum, além de boas práticas para avaliação dos seus DGA. 


\section{Procedimentos Metodológicos}

A metodologia utilizada possui uma abordagem qualitativa, através de uma pesquisa descritiva e exploratória, que fez uso da pesquisa documental em legislações sobre dados abertos. Como instrumentos foram utilizados o ranking Global Right to Information Rating, que reúne e avalia as legislações sobre acesso à informação de vários países do mundo; as legislações do México sobre dados abertos; e a métrica alternativa brasileira DGABr.

Com uma abordagem qualitativa, a pesquisa buscou identificar os países da América Latina que possuem legislação de acesso à informação e/ou política de dados abertos/DGA a fim de fazer possíveis adaptações da métrica brasileira para avaliar os DGA desses países. Para tanto, a pesquisa está dividida em três etapas, conforme explicitado no Quadro 1.

Quadro 1. Etapas da Pesquisa.

\begin{tabular}{lll}
\hline Etapa & \multicolumn{1}{c}{ Objetivo específico da pesquisa } & Técnica de pesquisa: Instrumentos/Procedimentos \\
\hline 1 & Identificar os países da América Latina que possuem legislação & Pesquisa documental: pesquisas na Internet e nos sites das \\
& sobre acesso à informação/política de dados abertos definida. & fundações e institutos sobre acesso à informação e dados abertos. \\
2 & Identificar as legislações mais abrangentes e com maiores & Pesquisa documental: análise das avaliações existentes no ranking \\
& critérios de avaliação dos DGA. & Global Right to Information Rating. \\
& Analisar a DGABr com base em outras legislações e propor & Pesquisa analítica: análise da legislação do México para dados \\
& adequações para sua utilização em outros países. & abertos e comparação com a DGABr. \\
\hline
\end{tabular}

Fonte: Elaborado pelas autoras (2019).

Nota: DGA: Dados Governamentais Abertos.

Na primeira etapa, foram realizadas buscas na Internet (Google e Google acadêmico) para identificar as legislações sobre o acesso à informação e dados abertos. Foram utilizados os descritores "dados abertos", "legislação" e "América Latina" nos idiomas português e inglês ("open data", "law", "Latin America"). Diante dos poucos resultados encontrados, com informações fragmentadas e direcionadas para sites não oficiais, optou-se por analisar o material publicado no estudo Global Right to Information Rating, o qual foi citado pela Organización de las Naciones Unidas para la Educación, la Ciencia y la Cultura (2017) no documento: "Acceso a la información: lecciones de la América Latina". O documento, além de ser citado em alguns resultados encontrados nas buscas, é atualizado, possui análises até 2018 e envolve mais de 15 países da América Latina.

A partir de um estudo realizado com vários países do mundo (Figura 1), foi criado o Right to Information Rating (RTI Rating), um programa fundado pelo Access Info Europe (AIE) e pelo Centre for Law and Democracy (CLD). A ideia central do RTI Rating é fornecer aos defensores, reformadores, legisladores e outros profissionais da área de Direito da Informação uma ferramenta confiável para avaliar comparativamente a força geral de uma estrutura legal para o acesso à informação.

O RTI Rating indica os pontos fortes e fracos do arcabouço legal e fornece um meio útil para localizar áreas que necessitam de melhorias. O Global Right to Information Rating avalia as legislações sobre o direito à informação em diversos países (Figura 1), porém, é importante destacar que o Global Right to Information Rating se limita a medir a estrutura legal e não mede a qualidade da implementação. Contudo, a estrutura legal é um pré-requisito para a plena implementação do direito à informação. A Figura 1 apresenta os países e a categoria obtida no ranking (Centre For Law And Democracy, 2018). O site da pesquisa disponibiliza a legislação de todos os países analisados e os resultados dos indicadores propostos no estudo. O Global Right to Information Rating é definido como: 
RTI

Global Right to Information

Rating Map

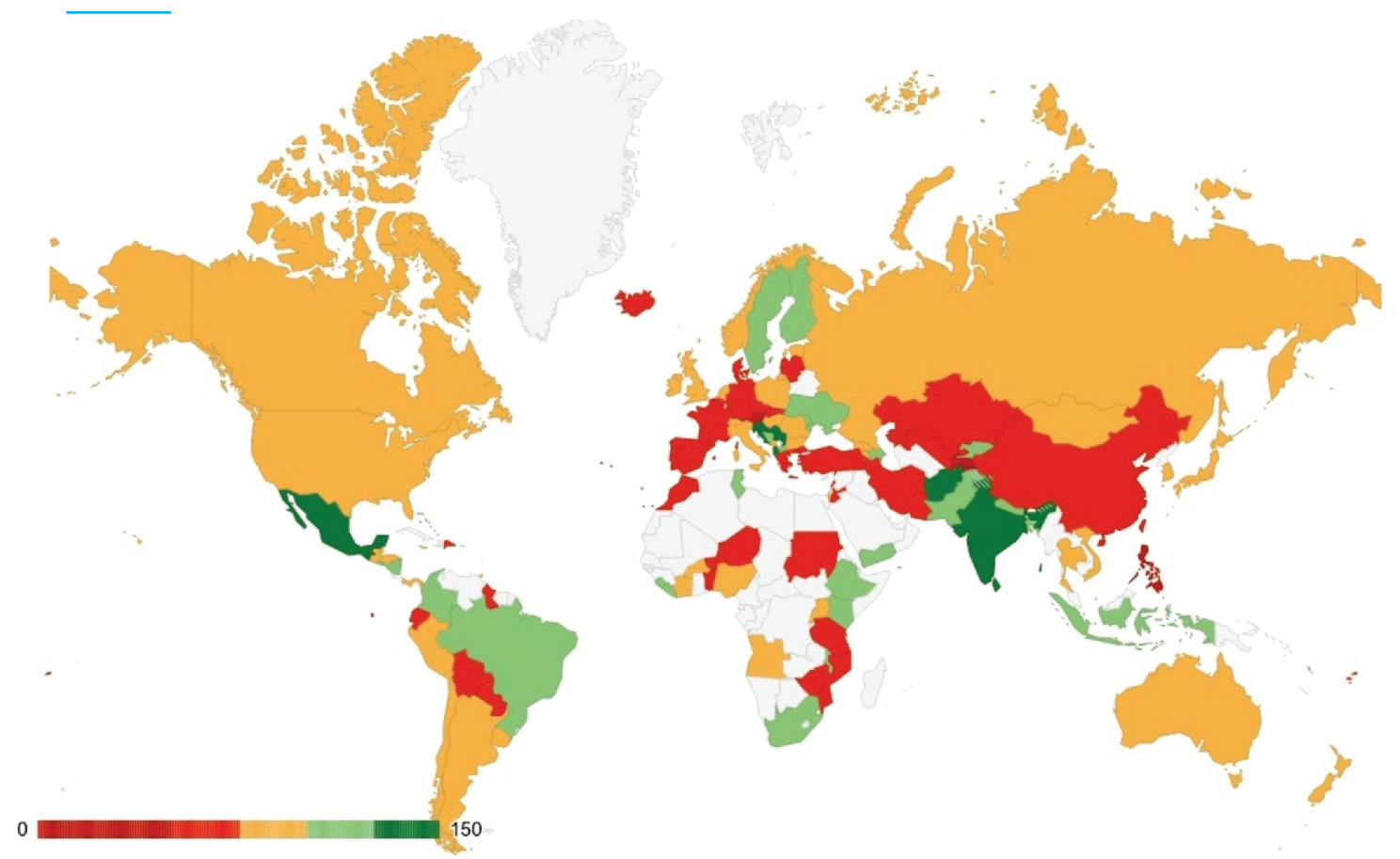

\begin{tabular}{l|l|l|l|l|}
{$[0-50]$} & {$[51-75]$} & {$[76-100]$} & {$[101-125]$} & {$[126-150]$}
\end{tabular}

RTI

\section{Country Rating Results}

Top 10

\begin{tabular}{|l|c|}
\hline Afghanistan & 139 \\
\hline Mexico & 136 \\
\hline Serbia & 135 \\
\hline Sri Lanka & 131 \\
\hline Slovenia & 129 \\
\hline India & 128 \\
\hline Albania & 127 \\
\hline Croatia & 126 \\
\hline Liberia & 124 \\
\hline El Salvador & 122 \\
\hline
\end{tabular}

Bottom 10

\begin{tabular}{|l|c|}
\hline Austria & 33 \\
\hline Palau & 33 \\
\hline Liechtenstein & 39 \\
\hline Philippines & 46 \\
\hline Tajikistan & 49 \\
\hline East Timor & 51 \\
\hline Benin & 52 \\
\hline Germany & 54 \\
\hline Jordan & 56 \\
\hline Taiwan & 57 \\
\hline
\end{tabular}

\section{(c) (i) (2)}

Figura 1. Países avaliados no Global Right to Information Rating.

Fonte: Centre for Law and Democracy (2018). 
[...] uma metodologia que avalia comparativamente a força das legislações de direito à informação em todo o mundo. No coração da metodologia para aplicar a avaliação de direito à informação estão 61 indicadores, cada um correspondendo a uma característica particular de um bom regime de direito à informação. Para cada Indicador, os países ganham pontos dentro de um intervalo definido de pontuações (na maioria dos casos 0-2), dependendo de quão bem o quadro legal fornece o indicador, para um total possível de 150 pontos. Os indicadores são divididos em sete categorias diferentes, a saber: Direito de Acesso, Escopo, Solicitação de Procedimentos, Exceções e Recusas, Apelações, Sanções e Proteções, e Medidas Promocionais (Centre For Law And Democracy, 2018, tradução nossa).

Considerando que o acesso à informação é o início para criação de políticas de dados abertos, o Global Right to Information Rating foi utilizado nesta pesquisa para identificar as legislações dos países da América Latina e compará-las, uma vez que o estudo já faz uma análise bem criteriosa dessas legislações. Dessa forma, na segunda etapa da pesquisa foram analisadas as pontuações atingidas no ranking pelos países da América Latina.

Os cinco países com melhor pontuação, nessa respectiva ordem, foram: México (136 pontos), El Salvador (122 pontos), Nicarágua (111 pontos), Brasil (108 pontos) e Colômbia (102 pontos). Optou-se por analisar a legislação mexicana para possíveis contribuições na métrica DGABr para América Latina, pois a referida métrica foi construída com base na legislação brasileira, ranqueada em quarto lugar; além disso, dos outros três países melhores pontuados, o México se destaca com a melhor pontuação na América, além de também ser indicado por alguns autores, como Balbino e Venancio (2017), por possuir uma legislação mais completa e detalhada que a brasileira e por ter publicada uma política de dados abertos.

Na terceira etapa da pesquisa, as dimensões da métrica DGABr foram analisadas com relação à legislação mexicana. Para tanto, inicialmente foi realizada a leitura da legislação disponibilizada no Global Right to Information Rating sobre acesso à informação e a política de dados abertos do México publicada inicialmente em 2015.

Para comparação com a DGABr, foi utilizado o quadro resumo (Quadro 2) da métrica e consultada a descrição correspondente para cada uma das dimensões e níveis disponibilizados no site da métrica: https:/www.dgabr.com.br/.

Ressalta-se que a construção da métrica DGABr é resultado de uma ampla pesquisa que considerou legislações, métricas, modelos e indicadores sobre dados abertos e DGA nos idiomas português, inglês e espanhol, entre 2009 e 2018. Também foram considerados padrões mundiais para publicação de dados abertos, como os oito

Quadro 2. Resumo da Métrica DGABr.

\begin{tabular}{|c|c|c|c|c|c|}
\hline \multirow{2}{*}{\multicolumn{2}{|c|}{ Dados Abertos }} & & Legal & \multirow{2}{*}{\multicolumn{2}{|c|}{ Gerencial }} \\
\hline & & D11 & Tipo de informação & & \\
\hline$\underset{\gg}{Z} \quad \mathrm{D} 1$ & Dados completos & D12 & Gestão de dados sensiveis & D21 & Priorização e Estratégias de abertura \\
\hline \begin{tabular}{|l|l|}
$\mathrm{D} 2$ \\
\end{tabular} & Dados primários & D13 & Plano de Dados Abertos & D22 & Estratégias de investimento e cronograma \\
\hline D3 & Dados atualizados & & Técnica & D23 & Manutenção e correção de problemas \\
\hline D4 & \begin{tabular}{|c|} 
Dados acessiveis \\
\end{tabular} & D14 & Fonte da informação & \multirow{2}{*}{\multicolumn{2}{|c|}{ Reúso }} \\
\hline D5 & Dados processáveis por máquina & D15 & Formato dos dados & & \\
\hline D6 & Acesso não discriminatório & D16 & Qualidade dos dados & D24 & Quantidade de conjuntos de dados publicados \\
\hline D7 & Dados não proprietários & D17 & Metadados & $\mathrm{D} 25$ & Quantidade de aplicativos desenvolvidos \\
\hline D8 & Dados livres de licença & D18 & Vocabulário & D26 & Quantidade de download dos aplicativos \\
\hline D9 & URLs desenhadas & D19 & Dimensão geográfica & D27 & Engajamento da sociedade civil \\
\hline D10 & Dados ligados & D20 & Catalogação dos dados & D28 & Informaçöes sobre o reúso de DGA \\
\hline
\end{tabular}

Fonte: Nascimento-Silva (2018a). 
princípios dos DGA (Open Gov Data, 2007) e o modelo das cinco estrelas proposto por Timothy John Berners-Lee (Silva, 2013). Legislações e publicações espanholas contribuíram demasiadamente para avaliação do reúso dos DGA, como a Comisión Europea (1998) e a Comisión Europea (2011). Destacam-se o projeto Aporta, que incentiva a abertura dos DGA e seu reúso desde 2009 (Aporta, 2019) e a Norma UNE 178301:2015 (Normalización Española, 2015), a qual traz aspectos relevantes sobre avaliação dos DGA (UNE 178301) que foram adaptados ao contexto brasileiro.

A métrica DGABr possui cinco perspectivas: Dados Abertos, Legal, Técnica, Gerencial e Reúso que incluem domínios importantes para a métrica. Cada perspectiva possui um peso, de 1 a 3, e é composta por dimensões de avaliações. Para as dimensões são atribuídos seis níveis, de 0 a 5, que correspondem, respectivamente, as seguintes classificações: Inexistente, Em construção, Não executado, Executado parcialmente, Resultados existentes e Resultados avançados. Ao todo, foram definidas 28 dimensões; e o somatório do nível obtido em cada dimensão multiplicado pelo peso da perspectiva resulta no indicador de potencial reúso dos DGA publicados (Nascimento Silva, 2018b).

Em uma prova de conceito realizada com a métrica DGABr, foram analisados conjuntos de dados do Ministério da Educação (MEC) - órgão da administração pública federal do Brasil -, através dos conjuntos de dados dos seguintes programas: Programa Universidade para Todos (ProUni), Programa Nacional de Acesso ao Ensino Técnico e Emprego (Pronatec) e Programa Mais Educação (PME). Na análise dos conjuntos de dados do MEC, foi possível identificar conjuntos de dados com uma pontuação alta, como o ProUni, e outros com pontuação mais baixa, como o PME. Dessa forma, a análise de vários conjuntos de dados permitiu observar essas diferenças e atingir um valor médio nas dimensões avaliadas, retratando a realidade do órgão quanto ao potencial reúso de seus DGA em um segmento específico. O MEC obteve o indicador 4 para os programas avaliados, o que representa um valor bom em uma escala de 1 a 5 (Nascimento Silva, 2018a).

Destaca-se que a métrica DGABr é versátil, uma vez que pode ser utilizada por atores da sociedade para avaliar conjuntos de dados publicados e também pode ser utilizada como um instrumento de autoavaliação para o próprio órgão que disponibilizou os dados. Para tanto, a avaliação deve considerar rigorosamente os níveis de avaliação de cada dimensão e avaliar elementos legais, gerenciais e técnicos que devem ser divulgados juntamente com os conjuntos de dados publicados.

\section{Resultados e Discussão}

Um dos fundamentos da métrica DGABr é a legislação sobre acesso à informação e à política de dados abertos vigente no Brasil. O Plano de Dados Abertos foi um dos documentos norteadores da métrica por incluir várias recomendações e exigências para abertura e publicação dos DGA no Brasil. No entanto, como a métrica também foi construída com base em padrões internacionais para publicação de DGA, com adaptações, espera-se que ela possa ser utilizada em outros países. Conforme o governo do México (Gobierno de México, 2017), as regulamentações relacionadas às iniciativas de dados abertos são: (1) Ley General de Transparencia y Acceso a la Información Pública Gubernamental (México, 2015c); (2) Decreto por el que se Establece la Regulación en Materia de Datos Abiertos (México, 2015a); (3) Guía de Implementación de la Política de Datos Abiertos na versão 2015 (México, 2015b) e 2017 (México, 2017a); (4) Norma Técnica para la Publicación de Datos Abiertos de Información Estadística y Geográfica de Interés Nacional (México, 2014); (5) Esquema de Interoperabilidad y Datos Abiertos (México, 2011).

Segundo o governo do México, o país destacou-se nos seguintes indicadores internacionais sobre dados abertos (México, 2018): (a) 10 Regional e $11^{\circ}$ geral do Barômetro de Dados Abertos 2017 da World Wide Web Foundation; (b) 2a Uso e impacto da The Organisation for Economic Co-operation and Development (OECD) 2017 Our Data Index; (c) $1^{\circ}$ Regional e $16^{\circ} \mathrm{global}$ no Open Data Inventory da Open Data Watch; (d) $2^{\circ}$ Regional e $11^{\circ}$ geral no índice de dados abertos do Open Knowledge International. 
Foram consultadas as cinco legislações listadas sobre dados abertos do México. O Guía de Implementación de la Política de Datos Abiertos é um documento que auxilia a cumprir todas as normas em relação à publicação de dados abertos no México, definindo os passos a serem realizados, prazos e procedimentos. A versão publicada em 12 de dezembro de 2017 substitui a versão inicial, publicada em 15 de junho de 2015. Por apresentar grande semelhança com o Plano de Dados Abertos, documento orientador da política de dados abertos do Brasil que foi utilizado na construção da métrica DGABr, o Guía de Implementación de la Política de Datos Abiertos, a partir de agora denominado como Guia, foi o documento utilizado para análise da política de dados abertos do México nesta pesquisa.

O Guia apresenta inicialmente as características mínimas para os dados abertos:

I. Livre: será obtido sem dar qualquer consideração em troca;

II. Não discriminatório: eles estarão acessíveis sem restrições de acesso para os usuários;

III Uso livre: citará a fonte de origem como o único requisito a ser usado livremente;

IV. Legíveis por máquinas: devem ser estruturados, total ou parcialmente, para serem processados e interpretados por equipamentos eletrônicos automaticamente;

V. Integrais: devem conter, na medida do possível, o assunto que descrevem em detalhes e com os metadados necessários;

VI. Primária: virá da fonte de origem com o maior nível de desagregação possível;

VII. Oportuno: será atualizado periodicamente, conforme forem gerados;

VIII. Permanente: deve ser mantido a tempo, para o qual, as versões históricas relevantes

(México, 2017, s/p, tradução nossa)

De acordo com a perspectiva Dados Abertos da métrica DGABr, pautada nos princípios dos DGA, que estabeleceram recomendações de melhores práticas sobre como os governos deveriam publicar os dados na internet (Open Gov Data, 2007), e no esquema das cinco estrelas, que tem como referência conceitos de dados abertos conectados(Open Knowledge Brasil, 2013), a legislação mexicana também destaca essas características básicas dos dados abertos, apresentando conformidade com a DGABr. Os quatro passos do Guia, subdivididos em 14 itens, foram comparados com as dimensões existentes na DGABr, conforme apresentado no Quadro 3.

No Item 1.1, a definição de responsáveis e a formação de um grupo de trabalho são destacadas no guia mexicano. Na Lei de acesso à informação, Lei no 12.527, de 2011 (Brasil, 2011), é prevista uma autoridade responsável, porém sem a divisão de tarefas e responsabilidades propostas nesse item. A DGABr não trata especificamente dos responsáveis e das respectivas funções, somente das estratégias de investimento e cronograma (D22: Estratégias de investimento e cronograma), porém é uma definição importante que poder ser incluída na legislação brasileira. Para a métrica não haveria essa necessidade, já que o foco é a publicação e o reúso dos DGA.

O item 1.2 é similar à dimensão D21: Priorização e estratégias de abertura. Porém, no plano de dados abertos - documento da legislação brasileira -, não há uma obrigatoriedade quanto aos itens que serão inclusos; essa avaliação é feita por cada órgão. O item 1.3 é similar à publicação do plano de dados abertos (D13: Plano de Dados Abertos), porém focaliza a atualização constante dele. Para esses três itens, não haveria necessidade de alteração na DGABr, já que a definição de responsáveis e o grupo de trabalho são questões gerenciais que não tratam diretamente dos dados abertos; além disso, os itens 1.2 e 1.3 já são verificados pela métrica. Assim, todos os três itens do passo 1 são atendidos pela métrica.

A formatação dos dados, tratada no item 2.1, é bem definida na legislação mexicana, e os formatos são explicitados no próprio Guia. Apesar de fragmentado em vários documentos da legislação brasileira, a formatação também foi um item definido na DGABr através da dimensão D15: Formato dos dados, que recomenda a utilização de formatos abertos conforme os padrões nacionais e internacionais para publicação dos dados abertos. Os padrões sugeridos pelo guia mexicano (padrão ISO 8601, formato UTF-8 para textos e padrão ASCII6) podem ser inclusos na DGABr como forma de complementação da métrica. 
Quadro 3. Guia de Implementação da Política de Dados Abertos.

\begin{tabular}{|c|c|c|}
\hline Atividade & Meios de verificação/Ações & Dimensão DGABr \\
\hline \multicolumn{3}{|c|}{ Passo 1 - Planejar } \\
\hline $\begin{array}{l}\text { 1.1 Formar um grupo de tra- } \\
\text { balho, designar um coor- } \\
\text { denador e um adminis- } \\
\text { trador de dados abertos. }\end{array}$ & Nomeação do grupo de trabalho. & $\begin{array}{l}\text { D22: Estratégias de investi- } \\
\text { mento e cronograma. }\end{array}$ \\
\hline 1.2 Priorizar dados de valor. & $\begin{array}{l}\text { A priorização dos dados deve incluir obrigatoriamente: 1. Os dados solicitados através } \\
\text { da Infraestrutura de Dados Abertos; } 2 \text {. Os dados dos registros públicos oficiais gerados } \\
\text { pela instituiçãa; } 3 \text {. Os dados públicos gerados ou geridos pelos sistemas de informação } \\
\text { da instituição, e 4. Dados públicos utilizados por ferramentas digitais, aplicações web } \\
\text { ou móveis, desenvolvidos pela instituição ou em seu nome. }\end{array}$ & $\begin{array}{l}\text { D21: Priorização e estraté- } \\
\text { gias de abertura. }\end{array}$ \\
\hline $\begin{array}{l}\text { 1.3 Gerar, publicar e atuali- } \\
\text { zar o Plano Institucional } \\
\text { para a publicação de da- } \\
\text { dos abertos. }\end{array}$ & $\begin{array}{l}\text { Publicação do Plano Institucional de Publicação de Dados Abertos em datos. } \\
\text { gob.mx. }\end{array}$ & $\begin{array}{l}\text { D13: Plano de Dados Aber- } \\
\text { tos. }\end{array}$ \\
\hline
\end{tabular}

Passo 2 - Publicar

2.1 Converter os dados em - Exportar os dados para arquivos em formato aberto formatos abertos.

- Identificar se é necessário estruturar formatos adicionais que facilitem sua leitura e uso de acordo com o tipo de dado;

- Garantir um formato válido e a estrutura do arquivo.

São recomendados formatos abertos: CSV, JSON ou XML, ODT, SQLite e para dados espaciais SHP, GeoJSON ou KML. Formatos adicionais podem ser publicados para cada conjunto de dados a fim de garantir maior inclusão e compreensão.

2.2 Aumentar a interopera- Interoperabilidade

bilidade e usabilidade - Utilizar o Marco Geoestatístico Nacional para dados publicados que contenham dos dados abertos. referências geográficas;

A DGABr avalia somente alguns formatos recomendados.

- Utilizar o formato UTF-8 para codificação de textos;

- Utilizar a norma ISO 8601;

- Utilizar o padrão ASCll6;

- Utilizar o padrão para geração e publicação de boletins e notificações técnicas:

- Utilizar as diretrizes do portal oficial de dados abertos do México (datos.gob.mx) e o Guia Anticorrupção de Dados Abertos da Carta Internacional de Dados Abertos (http://workspace.unpan.org/sites/Internet/Documents/UNPAN9 7913. pdf).

Usabilidade

Para aumentar a usabilidade dos dados abertos, as instituições devem: promover a legibilidade por máquina, promover a legibilidade por humanos e estabelecer categorias para os dados.

2.3 Melhorar a disponibilidade e os meios de distribuição dos dados abertos.

Publicar os dados abertos preferencialmente em serviços da Web e APIs, nos termos estabelecidos no esquema de interoperabilidade de dados abertos (EIDA): as instituições devem utilizar os seguintes meios de distribuição dos dados abertos: web Services, arquivos e feeds de notícias.

D15: Formato dos dados.

(n)

Garantir a disponibilidade das URLs para baixar os recursos de dados.

D20: Catalogação dos dados (em partes).

D15: Formato dos dados. D23: Manutenção e correção de problemas. 
Quadro 3. Guia de Implementação da Política de Dados Abertos.

\begin{tabular}{|c|c|c|}
\hline Atividade & Meios de verificação/Ações & Dimensão DGABr \\
\hline \multicolumn{3}{|c|}{ Passo 2 - Publicar } \\
\hline $\begin{array}{l}\text { 2.4 Documentar de acordo } \\
\text { com o padrão DCAT* e pu- } \\
\text { blicar no Catálogo Institu- } \\
\text { cional de Dados Abertos. }\end{array}$ & $\begin{array}{l}\text { Documentar os dados e publicar no catálogo institucional de dados abertos. } \\
\text { - Seguir o guia para documentar metadados na plataforma ADELA*; } \\
\text { - Utilizar dicionário de dados; } \\
\text { - Publicar dados abertos de acordo com a periodicidade estabelecida no Plano } \\
\text { Institucional de Publicação de Dados Abertos. }\end{array}$ & $\begin{array}{l}\text { D20: Catalogação dos dados. } \\
\text { D17: Metadados. } \\
\text { D18: Vocabulário. }\end{array}$ \\
\hline
\end{tabular}

Passo 3 - Aperfeiçoar

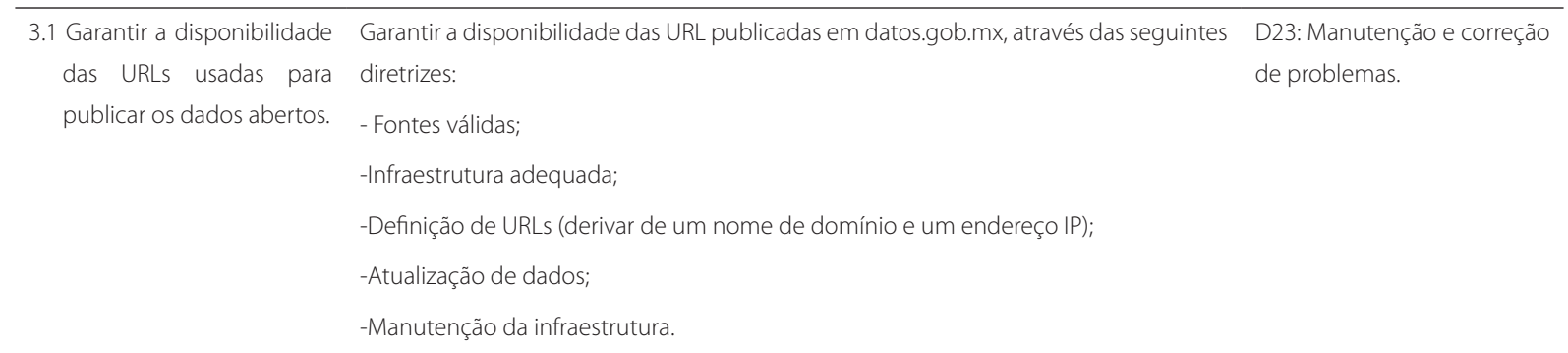

3.2 Fomentar a qualidade dos Recomendar as instituições sobre como aumentar a conformidade com a política de

D13: Plano de Dados Abertos. dados abertos publicados.

dados abertos e a qualidade e utilidade dos dados publicados.

D16: Qualidade dos dados.

- Recomendações gerais: conformidade com a política de dados abertos, solicitações

D23: Manutenção e correção de cidadãos, links quebrados e qualidade dos metadados, entre outros;

de problemas.

- Atividades pendentes: publicação ou atualização dos dados de acordo com o Plano Institucional de Publicação de Dados Abertos.

3.3 Responder as solicitações Responder aos pedidos dos cidadãos sobre dados abertos.

Não aplicável. dos cidadãos feitas no portal datos.gob.mx.

3.4 Garantir a conformidade Verificar a conformidade com as ações deste Guia e sua implementação. D13: Plano de Dados Abertos. com a política de dados abertos.

Passo 4 - Promover

4.1 Assegurar a geração, pu- As instituições que desenvolvam ou mantenham ferramentas ou aplicativos de blicação e uso de dados abertos em ferramentas e aplicações digitais. acesso público devem, obrigatoriamente:

D26: Quantidade de download dos aplicativos.

1. Publicar ou referenciar todos os dados gerados ou utilizados por eles em datos. gob.mx, de acordo com as disposições deste Guia;

2. Publicar a ferramenta ou aplicativo correspondente na seção Ferramentas e/ou na seção de Aplicativos do portal datos.gob.mx.

D28: Informações sobre o reúso de DGA.

3. Incluir um link visível na ferramenta ou aplicativo para baixar os dados de datos.gob.mx.

São consideradas ferramentas: Sites técnicos, Processadores analíticos (comumente relacionados ao "Business Intelligence", incluindo dashboards, calculadoras e/ou modelos numéricos), visualizadores geográficos, indexadores de dados (sobre bancos de dados, coleções de documentos, arquivos, digitalizações, áudio, vídeo ou imagens) e aplicações web ou mobile. 
Quadro 3. Guia de Implementação da Política de Dados Abertos.

\begin{tabular}{|c|c|c|}
\hline Atividade & Meios de verificação/Ações & Dimensão DGABr \\
\hline \multicolumn{3}{|c|}{ Passo 4 - Promover } \\
\hline \multirow[t]{3}{*}{$\begin{array}{l}\text { 4.2 Estimular o uso de dados } \\
\text { abertos pelos cidadãos. }\end{array}$} & $\begin{array}{l}\text { - Ter contato constante com todos os setores da sociedade para conhecer a opinião } \\
\text { direta dos usuários sobre a prioridade, estrutura, qualidade e uso dos dados } \\
\text { publicados em datos.gob.mx. }\end{array}$ & $\begin{array}{l}\text { D27: Engajamento da so- } \\
\text { ciedade civil. }\end{array}$ \\
\hline & $\begin{array}{l}\text { - Promover estratégias de consulta para gerar impacto através da publicação e } \\
\text { utilização de dados abertos em colaboração com os cidadãos. }\end{array}$ & \\
\hline & $\begin{array}{l}\text { - Realizar hackathons para incentivar uma maior participação cidadã para o desen- } \\
\text { volvimento de aplicações, plataformas do setor público, visualizações ou outras } \\
\text { soluções tecnológicas exigidas pelo governo. }\end{array}$ & \\
\hline \multirow[t]{3}{*}{$\begin{array}{l}\text { 4.3 Estabelecer estratégias de } \\
\text { comunicação digital. }\end{array}$} & $\begin{array}{l}\text { As instituições devem ter uma estratégia de comunicação digital, que se torna } \\
\text { essencial para conscientizar a população sobre a disponibilidade de novos recur- } \\
\text { sos de dados abertos, especialmente aqueles de alto valor. }\end{array}$ & $\begin{array}{l}\text { D27: Engajamento da so- } \\
\text { ciedade civil. }\end{array}$ \\
\hline & $\begin{array}{l}\text { - Informar os Links de Comunicação Digital sobre a publicação de Dados Abertos } \\
\text { de alto valor; }\end{array}$ & \\
\hline & $\begin{array}{l}\text { - Usar os mecanismos de comunicação disponíveis na instituição para comunicar } \\
\text { dados e/ou uso de dados abertos. }\end{array}$ & \\
\hline
\end{tabular}

Fonte: Adaptado de México (2017a, tradução nossa).

Nota: *Padrão internacional projetado para facilitar a interoperabilidade entre os catálogos de dados publicados na rede. Disponível em: http://www.w3.org/TR/ vocab-dcat/ ${ }^{* *}$ A plataforma digital para publicar no portal data.gob.mx e monitorar a conformidade com a Política de Dados Abertos em cada unidade, entidade ou empresa do Estado (México, 2017b, tradução nossa).

No item 2.2, que trata da interoperabilidade e usabilidade dos dados, a DGABr segue o Padrão de Interoperabilidade do Brasil (e-PING). Ademais, as recomendações do Guia mexicano também são sugeridas nos guias de publicação do Brasil. A usabilidade não é tratada especificamente na métrica, e, apesar de alguns itens de usabilidade estarem previstos na legislação brasileira, uma dimensão que trate especificamente da usabilidade pode ser interessante para a DGABr. Como os padrões de publicação de DGA no Brasil somente sugerem boas práticas e elas não são implementadas de maneira obrigatória, considerou-se que o item 2.2 não foi atendido pela DGABr.

A disponibilização dos dados abertos, tratada no item 2.3, sugere a distribuição por web services, arquivos e feeds de notícia. Na DGABr é avaliada a disponibilização dos dados no portal de dados abertos, local oficial indicado pela legislação brasileira através de arquivos, correspondendo as dimensões D20: Catalogação dos dados e D15: Formato dos dados, respectivamente. A dimensão D20 é atendida parcialmente, já que o Portal Brasileiro de Dados Abertos disponibiliza os conjuntos de dados somente em arquivos. A dimensão D15: Formato dos dados avalia os tipos de arquivos, incluindo web services. O feed de notícias não é avaliado na DGABr, porém pode ser avaliado em uma próxima versão da métrica. A garantia da disponibilização das URL é verificada na dimensão de D23: Manutenção e correção de problemas da DGABr.

A documentação dos dados e sua catalogação no portal de dados abertos, item 2.4, é avaliada pela DGABr conforme a legislação brasileira nas dimensões D20: Catalogação dos dados, D17: Metadados e D18: Vocabulário. A adaptação da DGABr ocorreria com o propósito de avaliar de acordo com o padrão de publicação do país analisado. Dos quatro itens do passo 2, um é atendido em partes (2.3), um não é atendido (2.2) e os dois restantes são atendidos pela métrica.

O item 3.1 aborda a garantia da disponibilidade das URL que é tratada pela dimensão D23: Manutenção e correção de problemas. Destaca-se que elementos da infraestrutura, detalhados nesse item, podem ser inclusos na DGABr que não faz a avaliação de todos esses elementos. O item 3.2 envolve questões legais em relação à 
conformidade com a política de dados abertos, à qualidade dos dados e à correção de possíveis erros, sendo todos avaliados pela DGABr (D13: Plano de Dados Abertos, D16: Qualidade dos dados e D23: Manutenção e correção de problemas). O item 3.3 aborda as respostas aos cidadãos, contudo, por se tratar de elementos da transparência ativa, não está relacionado na DGABr. O item 3.4, sobre a conformidade com a política de dados abertos, possui relação com a perspectiva legal e com o atendimento do plano de dados abertos já inclusos na DGABr. A conformidade com a legislação vigente é um dos pilares da métrica $D G A B r$, que é refletido na dimensão D13: Plano de Dados Abertos, a qual deverá ser adaptada conforme a legislação do país avaliado. Dos quatro itens do passo 3, três são atendidos e um não é aplicável.

O item 4.1, sobre garantir a geração, publicação e o uso de dados abertos, é um item muito importante no ciclo de vida dos dados abertos que é destacado pela legislação mexicana. Na legislação brasileira, não há obrigatoriedade de informar onde os dados são reusados. A DGABr avalia timidamente essa questão através dos aplicativos que utilizam DGA e das informações de reúso dos dados (D26: Quantidade de download dos aplicativos e D-28 Informações sobre o reúso de DGA), mas é necessária uma adaptação para incluir outras ferramentas, como sugerido na legislação do México. A promoção do uso dos dados abertos e suas estratégias de comunicação, item 4.2 e 4.3, são tratados na DGABr através da dimensão D-27 Engajamento da sociedade civil. Dos três itens do passo 4 , todos foram atendidos, mas destaca-se que a DGABr precisa ser otimizada em relação à avaliação do reúso dos dados.

A partir da análise comparativa realizada entre o Guia de Implementação da Política de Dados Abertos do México e as perspectivas e dimensões da métrica alternativa DGABr, foi observado que, dos 14 itens do guia mexicano, um item não é aplicável a avaliação de dados abertos (3.3), um item não é coberto pela $\mathrm{DGBr}(2.2)$ e um item é atendido parcialmente (2.3). Logo, dentre os 13 itens avaliados, 11 (84,6\%) são totalmente atendidos pela DGABr e, de forma geral, 12 itens (92,3\%) são atendidos entre parcial e total.

Observa-se que o Guia faz um controle de datas para cada uma das tarefas planejadas, permitindo um maior controle das atividades e cumprimento de prazos, o que não é visualizado na documentação brasileira e pode ser considerada uma boa prática. A dimensão da DGABr que mais se aproxima desse controle de datas é a D22: Estratégias de investimento e cronograma, onde os órgãos devem criar um cronograma para planejar a publicação dos DGA. O Quadro 4 reúne as adaptações necessárias na DGABr, identificadas na comparação realizada, para sua utilização na América Latina.

Quadro 4. Adaptações na Métrica DGABr.

\begin{tabular}{|c|c|}
\hline Perspectiva & Adaptações nas dimensões da métrica DGABr \\
\hline Dados Abertos & Sem alterações. \\
\hline Legal & $\begin{array}{l}\text { D13: Plano de dados abertos: considerar a existência de documentos similares que auxiliem no planejamento da publicação } \\
\text { dos DGA. }\end{array}$ \\
\hline \multirow[t]{5}{*}{ Técnica } & $\begin{array}{l}\text { D15 - Formato dos dados: além de verificar o formato dos arquivos avaliar se os DGA estão em conformidade aos padrões } \\
\text { internacionais: }\end{array}$ \\
\hline & - ISO 8601: padrão para representação de data e hora; \\
\hline & - Formato UTF-8 para textos: codificação de caracteres; \\
\hline & - Padrão ASCII6: codificação de caracteres. \\
\hline & - Criar uma dimensão específica para avaliar a usabilidade. \\
\hline Gerencial & D23: Manutenção e correção de problemas: avaliar elementos de infraestrutura e rede. \\
\hline Reúso & $\begin{array}{l}\text { D25: Quantidade de aplicativos desenvolvidos: alterar o nome da dimensão para: 'Quantidade de aplicativos ou ferramentas } \\
\text { desenvolvidas'e avaliar qualquer outra ferramenta ou serviço que utilize os DGA, quando essa informação for registrada, já que } \\
\text { na legislação brasileira, por exemplo, não há essa obrigatoriedade. }\end{array}$ \\
\hline
\end{tabular}

Fonte: Elaborado pelas autoras (2019). 


\section{Considerações Finais}

Métricas para DGA e dados abertos ainda são consideradas métrica alternativas por abordarem um tema recente com iniciativas em construção, cuja avaliação ainda é tímida pelos governos. Ademais, as escassas métricas existentes não possuem maturidade, pois foram utilizadas em poucos estudos e ainda não são oficializadas por instituições e/ou governos.

A métrica DGABr foi criada através de uma pesquisa acadêmica a fim de suprir uma lacuna existente na avaliação dos DGA publicados pelo governo brasileiro. Diante do resultado positivo da métrica, que foi construída com base em padrões e experiências internacionais sobre dados abertos, a proposta deste artigo foi avaliar se a DGABr poderia ser adaptada para o contexto da América Latina.

Após análise das legislações dos países da América Latina e do estudo elaborado pelo Global Right to Information Rating, definiu-se como referência, para comparação com a DGABr, a legislação de dados abertos do México através do Guia de Implementação da Política de Dados Abertos, publicado em 12 de dezembro de 2017.

Todas as dimensões (28) que compõem as cinco perspectivas da métrica DGABr foram analisadas juntamente com os quatro passos do Guia, subdivido em 14 itens a serem verificados na implementação da política de dados abertos do México. Identificou-se que as perspectivas e dimensões da DGABr abordam, em geral, 92\% das definições do Guia mexicano, o qual também aborda padrões internacionais e boas práticas de publicação.

Destaca-se que as adaptações provenientes do estudo realizado são relevantes e irão contribuir para completar e melhorar a métrica DGABr. Durante a análise da legislação mexicana, com destaque para o Guia, foi observado um grande detalhamento das atividades a serem realizadas e a definição de prazos para todas elas. Esse detalhamento contribui para a implementação da política de dados abertos, seu controle e avaliação, como importante subdomínio das políticas de informação. A legislação mexicana ainda indica a utilização de padrões, detalhando os itens onde eles devem ser empregados, permitindo, assim, o controle e a mensuração, de forma objetiva, dos dados publicados. Na legislação brasileira, onde a política de dados abertos é fragmentada e existem diferentes recomendações para a publicação de DGA, a avaliação é dificultada.

A DGABr, em sua primeira versão, tem o objetivo de avaliar os DGA brasileiros e, com adaptações, poderá ser utilizada em outros países da América Latina. A métrica ainda é recente, assim, contribuições advindas de experiências e boas práticas na publicação de dados abertos serão sempre bem-vindas para o aperfeiçoamento da métrica. Como trabalho futuro, sugere-se a complementação da métrica DGABr em sua próxima versão, com as boas práticas e padrões existentes na legislação mexicana e a elaboração de uma derivação da métrica para ser utilizada na América Latina. Sugere-se, inclusive, uma nova nomenclatura: DGALat.

\section{Colaboradores}

Todos os autores contribuíram igualmente na concepção e desenho do estudo, coleta e análise de dados e redação final.

\section{Referências}

Aporta. Acerca de la iniciativa España: Aporta. Aporta, 2019 Disponible en: https://datos.gob.es/es/acerca-de-lainiciativa-aporta\#aporta. Acceso en: 15 jul. 2019.

Balbino, G.M.S.; Venancio, R.P. O acesso à informação pública: um estudo comparativo entre as legislações do Brasil e do México. Revista Ágora: Políticas Públicas e Comunicação, Gover- nança Informacional, v.1, n.Esp., p.4-18, 2017. Disponível em: https://seer.ufmg.br/index.php/revistaagora/article/view/ 6897. Acesso em: 10 jan. 2019.

Brasil. Presidência da República. Lei n¹2.527, de 18 de novembro de 2011. Regula o acesso a informações previsto no inciso XXXIII do art. 5o, no inciso II do § 30 do art. 37 e no 
§ 20 do art. 216 da Constituição Federal; altera a Lei no 8.112, de 11 de dezembro de 1990; revoga a Lei no 11.111, de 5 de maio de 2005, e dispositivos da Lei no 8.159, de 8 de janeiro de 1991; e dá outras providências. Diário Oficial da União, Brasília, 18 nov., 2011. Disponível em: http://www.planalto. gov.br/ccivil_03/_ato2011-2014/2011/lei/l12527.htm. Acesso em: 22 jan. 2019.

Brasil. Governo Federal. Lei Modelo de Acesso à Informação: qual a sua importância e por que está sendo revisada? Ouvidorias, 2018. Disponível em: http://www.ouvidorias.gov. br/noticias/lei-modelo-de-acesso-a-informacao. Acesso em: 10 jan. 2018.

Centre for Law and Democracy. Global right to information rating. Halifax: Centre for Law and Democracy, 2018. Available from: https://www.rti-rating.org/. Cited: Jan. 10, 2019.

Chile. Ministerio Secretaría General de la Presidencia. Ley 20285 para la transparencia de las oficinas públicas y acceso a la información estatal. Santigago: Congreso Nacional de Chile, 2008 Disponible en: https://www.leychile.cl/ Navegar?idNorma=276363. Acceso en: 15 jul. 2019.

Comisión Europea. La información del sector público: un recurso clave para Europa: Libro Verde sobre la información del Sector Público en la Sociedad de la Información. 1998. Disponible en: https:/gobiernoabierto.navarra.es/sites/default/files/opendata/ libro_verde_informacion.pdf. Acceso em: 7 mayo. 2019.

Comissão Europeia. Comunicação da Comissão ao Parlamento Europeu, ao Conselho, ao Comité Económico e social europeu e ao Comité das Regiões: dados abertos: um motor de inovação, crescimento e governação transparente. Bruxelas, 2011. Disponível em: https://eur-lex.europa.eu/LexUriServ/ LexUriServ.do?uri=COM:2011:0882:FIN:PT:PDF. Acesso em: 7 maio 2019.

Gobierno de México. Normatividad de Datos Abiertos en México. Ciudad del Mexico: México Digital, 2017. Disponible en: https:/datos.gob.mx/blog/normatividad-de-datos-abiertosen-mexico?category=gobernanza\&tag=nula. Acceso en: 7 maio 2019.

Gobierno de México. Acuerdo por el que se establece la guía de implementación de la Política de Datos Abiertos. Ciudad del Mexico: México Digital, 2018. Disponible en: https:// www.gob.mx/mexicodigital/articulos/acuerdo-por-el-quese-establece-la-guia-de-implementacion-de-la-politica-dedatos-abiertos. Acceso en: 10 jan. 2019.

Kalampokis, E.; Tambouris, E.; Tarabanis, K. A classification scheme for open government data: Towards linking decentralised data. International Journal of Web Engineering and Technology, v.6, n.3, p.266-285, 2011. Available from: https://dl.acm.org/citation.cfm?id=1999591. Cited: Jan. 10, 2019.

Lowry, J. Open government data literature review project. London: Department of Information Studies University College, 2015. Available from: https://interparestrust.org/assets/ public/dissemination/EU02_20151210_OpenGovernment DataLiteratureReview_FinalReport.pdf. Cited: Jan. 10, 2019.

México. Secretaria de Governación. Acuerdo por el que se establece el Esquema de Interoperabilidad y de Datos
Abiertos de la Administración Pública Federal. Diário Oficial de la Federacion, 2011. Disponible en: http://dof.gob.mx/nota_ detalle.php?codigo $=5208001 \&$ fecha $=06 / 09 / 2011$. Acceso en: 10 jan. 2019.

México. Secretaria de Governación. Acuerdo por el que se aprueba la Norma Técnica para el acceso y publicación de Datos Abiertos de la Información Estadística y Geográfica de Interés Nacional. Diário Oficial de la Federacíon, 2014. Disponible en: http://www.dof.gob.mx/nota_detalle.php? codigo $=5374183 \&$ fecha=04/12/2014. Acceso en: 10 jan. 2019 .

México. Secretaria de Governación. Decreto por el que se establece la regulación en materia de Datos Abiertos. Diário Oficial de la Federacion, 2015a. Disponible en: http://www. dof.gob.mx/nota_detalle.php?codigo $=5382838 \&$ fecha $=$ 20/02/2015. Acceso en: 10 jan. 2019.

México. Secretaria de Governación. Guía de implementación de la política de datos abiertos. Diário Oficial de la Federacion, 2015b. Disponible en: http://www.dof.gob.mx/nota_detalle. php? codigo $=5397117 \&$ fecha $=18 / 06 / 2015$. Acesso em: 10 jan. 2019.

México. Secretaria de Governación. Ley general de transparencia y acceso a la información pública. Diário Oficial de la Federacion, 2015c. Disponible en: http://www.dof.gob. mx/nota_detalle.php?codigo $=5391143 \&$ fecha $=04 / 05 / 2015$. Acceso en: 10 jan. 2019.

México. Secretaria de Governación. Guía de Implementación de la política de datos abiertos. Diário Oficial de la Federacion, 2017. Disponible en: http://dof.gob.mx/nota_detalle.php? codigo $=5507476 \&$ fecha=12/12/2017. Acceso en: 10 jan. 2019 .

Nascimento Silva, P.N. Dados governamentais abertos: métricas e indicadores de reuso. 2018. 322f. Tese (Doutorado em Gestão e Organização do Conhecimento) - Universidade Federal de Minas Gerais, Belo Horizonte, 2018a. Disponível em: http:// www.bibliotecadigital.ufmg.br/dspace/handle/1843/BUBDAYNG4U. Acesso em: 10 jan. 2019.

Nascimento Silva, P.N. DGABr: Métrica Brasileira. Belo Horizonte: Universidade Federal de Minas Gerais, 2018b. Disponível em: https://www.dgabr.com.br/. Acesso em: 15 jan. 2019.

Nascimento Silva, P.N.; Kerr Pinheiro, M.M. Métricas para dados governamentais abertos: análise de modelos internacionais. In: Encontro Nacional de Pesquisa em Ciência da Informação, 18., 2017, Marília. Anais [...] Disponível em: http://enancib. marilia.unesp.br/index.php/xviiienancib/ENANCIB/paper/ viewFile/90/525. Acesso em: 10 jan. 2019.

Nascimento Silva, P.N.; Kerr Pinheiro, M.M. Métricas para Dados Governamentais Abertos. RICl: Revista Ibero-Americana de Ciência da Informação, v.11, n.1, p.314-332, 2018. Disponível em: http://periodicos.unb.br/index.php/RICl/article/view/ 8431/7025. Acesso em: 07 jan. 2019.

Normalización Española. UNE 178301;2015: Ciudades Inteligentes: datos abiertos (Open Data). Madrid: AENOR, 2015. Disponible en: https://www.aenor.com/normas-ylibros/buscador-de-normas/UNE?c=N0054318. Acceso en: 7 mayo 2019.

Open Gov Data. 8 principles of open government data: Public resource. Sebastopol: OGD, 2007. Available from: https:// public.resource.org/8_principles.html. Cited: Jan. 10, 2019. 
Organización de las Naciones Unidas para la Educación, la Ciencia y la Cultura. Acceso a la información: lecciones de la América Latina. Montevideo: Unesco, 2017. Disponible em: https://unesdoc.unesco.org/ark:/48223/pf0000249837_spa. Aceso en: 2 jan. 2019

Perlingeiro, R.; Díaz, l.; Liani, M. Princípios sobre o direito de acesso à informação oficial na América Latina. Revista de Investigações Constitucionais, v.3, n.2, p.143-197, 2016. Disponível em: https://revistas.ufpr.br/rinc/article/view/464 51/28765. Acesso em: 10 jan. 2019.
Silva, N. Maturidade em Dados Abertos: entenda as 5 estrelas. [s.n.]: Open Knowledge Brasil, 2013. Disponível em: https:// br.okfn.org/2013/01/17/maturidade-em-dados-abertosentenda-as-5-estrelas/. Acesso em: 10 jan. 2019.

Simón, L.F R. et al. De la reutilización de la información del sector público a los portales de datos abiertos en Europa. Bid: Textos Universitaris De Biblioteconomia i Documentació, n.29, 2012. Disponible en: http://bid.ub.edu/29/ramos2.htm. Acceso en: 10 jan. 2019.
15

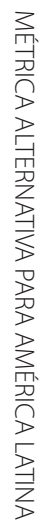

